\title{
Neurology
}

\section{Ataxin-1 and ataxin-2 intermediate-length PolyQ expansions in amyotrophic lateral sclerosis}

Francesca L. Conforti, Rossella Spataro, William Sproviero, et al.

Neurology 2012;79;2315; Published online before print November 28, 2012;

DOI 10.1212/WNL.0b013e318278b618

This information is current as of December 11, 2012

The online version of this article, along with updated information and services, is located on the World Wide Web at:

http://www.neurology.org/content/79/24/2315.full.html

Neurology ${ }^{\circledR}$ is the official journal of the American Academy of Neurology. Published continuously since 1951 , it is now a weekly with 48 issues per year. Copyright (C) 2012 by AAN Enterprises, Inc. All rights reserved. Print ISSN: 0028-3878. Online ISSN: 1526-632X.

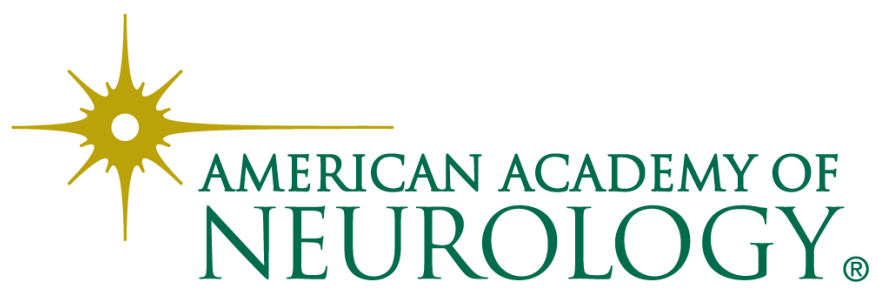


Francesca L. Conforti,

$\mathrm{PhD}$

Rossella Spataro, MD

William Sproviero, PhD

Rosalucia Mazzei, PhD

Francesca Cavalcanti, MD

Francesca Condino, $\mathrm{PhD}$

Isabella L. Simone, MD

Giancarlo Logroscino, MD

Alessandra Patitucci, PhD

Angela Magariello, PhD

Maria Muglia, PhD

Carmelo Rodolico, MD

Paola Valentino, MD

Francesco Bono, MD

Tiziana Colletti, BSc

Maria R. Monsurrò, MD

Antonio Gambardella, MD

Vincenzo La Bella, MD, $\mathrm{PhD}$

Correspondence \& reprint requests to Dr. La Bella: vincenzo.labella@unipa.it

\section{Ataxin-1 and ataxin-2 intermediate-length PolyQ expansions in amyotrophic lateral sclerosis}

\section{ABSTRACT}

Objective: Recent evidence suggests that intermediate-length polyglutamine (PolyQ) expansions in the ataxin-2 (ATXN-2) gene are a risk factor for amyotrophic lateral sclerosis (ALS). This work was undertaken with the aim to investigate the frequency of ataxin-1 (ATXN-1) and ATXN-2 PolyQ expansions in a cohort of patients with sporadic ALS (sALS) and patients with familial ALS (fALS) from southern Italy.

Methods: We assessed the PolyQ lengths of ATXN-1 and ATXN-2 in 405 patients with sALS, 13 patients with fALS, and 296 unrelated controls without history of neurodegenerative disorders.

Results: We found significantly higher intermediate PolyQ expansions $\geq 32$ for ATXN-1 alleles and $\geq 28$ for ATXN-2 alleles in the sALS cohort (ATXN-1: ALS, 7.07\% vs controls, 2.38\%; $p=0.0001$; ATXN-2: ALS, $2.72 \%$ vs controls, $0.5 \% ; p=0.001$ ). ATXN-1 CAT and ATXN-2 CAA interruptions were detected in patients with ALS only. Age at onset, site of onset, and sex were not significantly related to the ATXN-1 or ATXN-2 PolyQ repeat length expansions.

Conclusions: Both ATXN-1 and ATXN-2 PolyQ intermediate expansions are independently associated with an increased risk for ALS. Neurology ${ }^{\circledR} 2012 ; 79: 2315-2320$

\section{GLOSSARY}

ALS = amyotrophic lateral sclerosis; ATXN = ataxin; $\mathbf{f A L S}=$ familial amyotrophic lateral sclerosis; $\mathbf{N C}=$ normal control; $\mathbf{P Q B P}=$ PolyQ binding protein; PolyQ = polyglutamine; $\mathbf{R O C}=$ receiver operating characteristic; $\mathbf{s} \mathbf{A L S}=$ sporadic amyotrophic lateral sclerosis; $\mathbf{S C A}=$ spinocerebellar ataxia.

Amyotrophic lateral sclerosis (ALS) is a relentlessly progressive neurodegenerative disorder of motor neurons, leading to a severe muscle weakness and atrophy. ${ }^{1}$ Several mutated genes (e.g., Cu/Zn SOD1, FUS/TLS, TARDBP, C9ORF72) have been demonstrated to be implicated in the disease. $^{1-4}$

A recent work demonstrated that TDP-43, a TARDBP gene product, and ataxin-2 (ATXN-2) form a complex that depends on RNA binding and that a small number of patients with ALS are carriers of $A T X N-2$ intermediate expansions (27-33 glutamines). ${ }^{5}$ This finding led to a number of studies from America, Europe, and China that have now demonstrated that $A T X N-2$ intermediate poly-CAG expansions with CAA interruptions are indeed a risk factor for ALS. ${ }^{5-12}$ This effect appears to be specific, as ATXN-2 repeat length intermediate expansions in Alzheimer disease, Parkinson disease, and frontotemporal degeneration were not significantly more frequent than in controls. ${ }^{13}$

Clinical signs and symptoms of motor neuron degeneration, with bulbar and distal neurogenic muscle atrophy, have been described in spinocerebellar ataxias. ${ }^{14-16}$ In particular, the protein product of spinocerebellar ataxia 1 (SCA1), ataxin-1 (ATXN-1), forms aggregates in the nucleus and binds to coiled bodies, exerting a toxic effect on RNA metabolism, thus leading to neuron degeneration including motor neurons. ${ }^{17-19}$ These data point to a specific role of ataxin-1 protein in

\footnotetext{
From the Institute of Neurological Sciences (F.L.C., W.S., R.M., F. Cavalcanti, F. Condino, A.P., A.M., M.M., A.G.), National Research Council, Mangone, Cosenza; ALS Clinical Research Center (R.S., T.C., V.L.B.), Department of Experimental BioMedicine and Clinical Neurosciences, University of Palermo, Palermo; Institute of Neurology (W.S., P.V., F.B., A.G.), University Magna Graecia, Catanzaro; Department of Neurological and Psychiatric Sciences (I.L.S., G.L.), University of Bari, Bari; Department of Neurosciences (C.R.), Psychiatric and Anaesthesiological Sciences, University of Messina, Messina; and Second Division of Neurology (M.R.M.), Second University of Naples, Naples, Italy.

Go to Neurology.org for full disclosures. Disclosures deemed relevant by the authors, if any, are provided at the end of this article.
} 
motor neuron survival. The evidence that patients with SCA1 during the course of the disease can develop motor neuron degeneration is a further support to this hypothesis.

In this work, we analyzed the $A T X N-1$ and $A T X N-2$ polyglutamine (PolyQ) repeat length in a large cohort of patients with sporadic ALS (sALS) and patients with familial ALS (fALS).

METHODS Patients and controls. With written informed consent, blood samples were obtained from 405 patients diagnosed with sALS (236 male and 169 female; mean age at onset 57.07 years, SD 12.5) and 13 patients diagnosed with fALS (6 male and 7 female; mean age at onset 57.85 years, SD 11.9). All patients, diagnosed according to the El Escorial revised criteria, ${ }^{20}$ were previously screened for the presence of pathogenic mutations in the superoxide dismutase (SODI), TAR DNA-binding protein (TDP-43), angiogenin $(A N G)$, and fused in sarcoma/ translocated in liposarcoma (FUS/TLS) genes.

The very recent discovery of the C9ORF72 gene prompted us to search for the presence of the pathogenic GGGGCC expansion, by a repeat-method PCR assay (pathologic expansion $\geq 30$ repeats), in all patients (fALS and sALS) carrying an $A T X N-1$ or $A T X N-2$ intermediate PolyQ repeat length expansion. However, the examined cohort did not show any pathogenic expansion for this gene (data not shown).

A cohort of 296 geographically matched unrelated Italian individuals (171 male, 125 female; mean age 60.5 years, SD 15.6) without history of neurodegenerative disease were used as controls.

Standard protocol approvals, registrations, and patient consents. We received approval for these studies from the ethical standards institutional committees of each participating institution. A written consent was obtained by each subject who contributed a DNA sample for this study (consent for research).

Determination of $A T X N-2$ and $A T X N-1$ CAG repeat size. DNA was extracted from venous blood tissue using standard methods. Genotyping of the ataxin-2 and ataxin-1 CAG repeat number was performed using fluorescent-labeled primer PCR with capillary electrophoresis on an ABI3130xl sequencer (primer sequences are available on request) and analyzed with

\begin{tabular}{|c|c|c|c|c|c|}
\hline \multirow[t]{2}{*}{ Table 1} & \multicolumn{5}{|c|}{$\begin{array}{l}\text { Demographic and clinical characteristics of the patients with } \\
\text { amyotrophic lateral sclerosis (sporadic and familial) and controls }\end{array}$} \\
\hline & & sALS $(n=405)$ & fALS ( $n=13)$ & Controls $(n=296)$ & $p$ Value $^{a}$ \\
\hline Men, n (\%) & & $236(58.3)$ & $6(46.2)$ & $171(57.8)$ & $0.684^{b}$ \\
\hline Age at onse & $y$, mean $\pm S D$ & $57.07 \pm 12.5$ & $\begin{array}{l}57.85 \pm \\
11.9\end{array}$ & - & $0.970^{c}$ \\
\hline \multicolumn{6}{|c|}{ Site of symptom onset, $n(\%)^{d}$} \\
\hline Bulbar & & $62(18.7)$ & $2(15.4)$ & & $1.0^{\mathrm{e}}$ \\
\hline Spinal & & 266 (80.1) & 11 (84.6) & & \\
\hline
\end{tabular}

Abbreviations: $\mathrm{fALS}=$ familial amyotrophic lateral sclerosis; $\mathrm{sALS}=$ sporadic amyotrophic lateral sclerosis.

a $p$ Value refers to a comparison among 3 groups for sex and 2 groups for the other variables.

${ }^{\mathrm{b}} \chi^{2}$ test.

cMann-Whitney $U$ test.

${ }^{d}$ Site of symptom onset data were not available for 73 sALS cases.

${ }^{\mathrm{e}}$ Fisher exact test.
GeneMapper software version 4.0 (Applied Biosystems). A control subject with a 22/40 heterozygous genotype checked by direct sequencing was used as a calibrator. Expansions above 30 repeats were confirmed by a second analysis and directly sequenced after gel separation. The same fragments were also cloned into a pGEM-T Easy vector system I (Promega, Madison, WI) and sequenced bidirectionally in at least 3 independent clones (Applied Biosystems) to further verify the number of CAGs and the presence of interruptions (CAA/CAT).

Statistical analysis. The difference in sex distribution among groups was evaluated with the $\chi^{2}$ test. Kruskal-Wallis test was performed to compare age at examination and Mann-Whitney $U$ test was used for age at onset.

To determine the best cutoff to discriminate ALS cases from controls, a receiver operating characteristic (ROC) analysis was performed. We assign the same importance to the sensitivity and the specificity, so the optimal cutoff level was considered to be the value that had the highest unweighted sum of sensitivity and specificity values. $\chi^{2}$ test and Fisher exact test (when the smallest expected frequency was less than 5) were used to calculate the significance for the genetic association of ATXN-2/ATXN-1 repeat lengths and ALS. Odds ratios and $95 \%$ confidence intervals were calculated according to a logistic regression model adjusted for age and sex. Exact logistic regression model was used when the expected frequencies were low. In all tests, a $p$ value below 0.05 was considered significant.

In order to evaluate the differences in clinical and demographic characteristics between patients carrying and not carrying long $A T X N-2 / A T X N-1$ repeats, we used $\chi^{2}$ test (or Fisher exact test, as appropriate) for categorical variables and Mann-Whitney $U$ test for continuous variables.

Statistical analysis was performed in SPSS (version 17, for Windows). The exact logistic regression model was obtained by using the elrm package (version 1.2.1) implemented in $R$.

RESULTS We evaluated the $A T X N-1$ and $A T X N-2$ PolyQ repeat length in genomic DNA from 405 patients with sALS, 13 patients with fALS, and 296 neurologically normal controls (NC) from a relatively large Mediterranean area (southern Italy).

Table 1 summarizes the demographic and clinical characteristics of the 3 groups. All patients with ALS (both sporadic and familial) included in this study were diagnosed according to the World Federation of Neurology-El Escorial revised criteria. ${ }^{20}$ Mean age at onset was $57.07 \pm 12.5$ years for sALS and $57.85 \pm$ 11.9 years for fALS ( $p=0.970$, Mann-Whitney $U$ test). The M/F ratio was 1.39 for sALS, 0.85 for fALS, and 1.37 for NC $(p=0.684)$. Onset was bulbar in $18.7 \%$ of sALS and $15.4 \%$ of fALS cases.

The distribution of $A T X N-1$ and $A T X N-2$ PolyQ repeat length in sALS and $\mathrm{NC}$ are shown in the figure and table 2. We found that 57 out of the 806 ATXN-1 alleles in the sALS cohort harbored a $\geq 32$ PolyQ repeat length (7.07\%), as compared to 13 (2.38\%) out of the $544 \mathrm{NC}$ alleles $\left(p=0.0001, \chi^{2}\right.$ test; figure, A). For $A T X N-2$, a $\geq 28$ PolyQ repeat length was found in $22(2.72 \%)$ of the 808 sALS alleles and in only $3(0.5 \%)$ of the $586 \mathrm{NC}$ alleles 


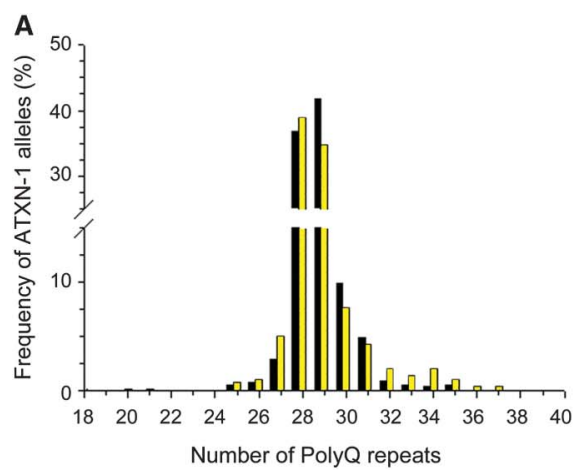

ATXN $=$ ataxin; Poly $Q=$ polyglutamine.

$\left(p=0.001, \chi^{2}\right.$ test; figure, B). Furthermore, both $A T X N-1$ and $A T X N-2$ intermediate PolyQ repeat length expansions are likely to be independently associated with an increased risk for sALS. Only 1 patient with ALS out of the 418 sALS and fALS patients and $296 \mathrm{NC}$ tested was found to be a carrier of both $A T X N-1$ and $A T X N-2$ allele expansions.

The analysis of $A T X N-1$ and $A T X N-2$ intermediate PolyQ repeat length expansions in fALS revealed that $A T X N-1$ might be a potential risk factor also in these patients (table 2). However, further studies with a larger patient cohort are needed to verify this association as the sample size was relatively small.

\begin{tabular}{|c|c|c|c|c|}
\hline Table 2 & \multicolumn{4}{|c|}{$\begin{array}{l}\text { Frequency of } \geq 32 A T X N-1 \text { and } \geq 28 A T X N-2 \text { intermediate PolyQ repea } \\
\text { length expansions in sALS and } f A L S\end{array}$} \\
\hline Alleles & sALS & Controls & $p$ Value & OR $(95 \% \mathrm{Cl})^{a}$ \\
\hline \multicolumn{5}{|l|}{ ATXN-1, n (\%) } \\
\hline$\geq 32$ repeats & $57(7.07)$ & $13(2.4)$ & $0.0001^{b}$ & $2.396(1.26-4.56)$ \\
\hline$<32$ repeats & 749 (92.9) & 531 (97.6) & & \\
\hline \multicolumn{5}{|l|}{ ATXN-2, n (\%) } \\
\hline$\geq 28$ repeats & $22(2.7)$ & $3(0.5)$ & $0.001^{b}$ & $5.832(1.71-9.78)$ \\
\hline \multirow[t]{2}{*}{$<28$ repeats } & 786 (97.3) & 583 (99.5) & & \\
\hline & fALS & Controls & $p$ Value & OR $(95 \%$ CI) \\
\hline \multicolumn{5}{|l|}{ ATXN-1, n (\%) } \\
\hline$\geq 30$ repeats & 8 (33.3) & 94 (17.3) & $0.056^{c}$ & $1.56(0.42-9.67)$ \\
\hline$<30$ repeats & $16(66.7)$ & $450(82.7)$ & & \\
\hline \multicolumn{5}{|l|}{ ATXN-2, n (\%) } \\
\hline$\geq 22$ repeats & $26(100)$ & 574 (97.9) & $1.0^{c}$ & NA \\
\hline$<22$ repeats & $0(0.0)$ & 12 (2.1) & & \\
\hline
\end{tabular}

Abbreviations: $\mathrm{ATXN}=$ ataxin; $\mathrm{Cl}=$ confidence interval; $\mathrm{fALS}=$ familial amyotrophic lateral sclerosis; NA = not available; $\mathrm{OR}=$ odds ratio; PolyQ = polyglutamine; $\mathrm{sALS}=$ sporadic amyotrophic lateral sclerosis.

${ }^{a}$ ORs and $95 \% \mathrm{Cls}$ were calculated according to a logistic regression or exact logistic regression model, as appropriate, adjusted for age and sex.

${ }^{b} \chi^{2}$ test.

${ }^{\mathrm{c}}$ Fisher exact test.

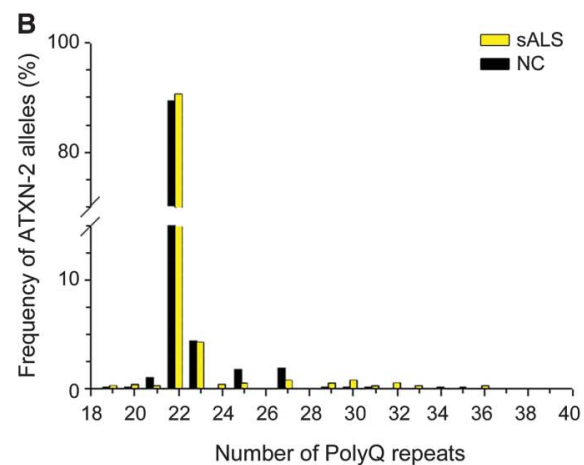

We then determined which demographic or clinical variable would have been associated with the $A T X N-1$ or $A T X N-2$ PolyQ repeat length expansions. We could only obtain information about sex, age at onset, and site of onset. Other clinical and demographic variables were not available at the time of this study. As shown in table 3, none of the variables tested in sALS and fALS were significantly related to the $A T X N-1$ and $A T X N-2$ PolyQ repeat length expansions. Direct DNA sequencing of the longest repeat alleles (i.e., both $A T X N-1$ and $A T X N-2, \geq 35$ and $\geq 31$ repeats, respectively) showed $A T X N-1$ CAT and $A T X N-2$ CAA interruptions in patients with ALS only.

DISCUSSION In the present study, we have evaluated the role of $A T X N-1$ and $A T X N-2$ expansions in a cohort of patients with ALS, and found a significant association of both $A T X N-1$ alleles $\geq 32$ and $A T X N-2$ alleles $\geq 28$ PolyQ repeats with sALS.

Both $A T X N-1$ and $A T X N-2$ expansions were detected in 1 patient with ALS only, and this strongly suggests that the 2 genetic variables are in fact independently related to an increased risk of ALS.

Our data confirm the recent reports that ATXN-2 is a genetic risk factor for ALS. ${ }^{5-12}$ The finding that $A T X N-1$ PolyQ repeats $\geq 32$ also represent a risk factor for ALS is novel, and suggests that intermediate expansions of both ataxins might indeed be involved in the pathogenesis of this neurodegenerative disorder.

$A T X N-2$ was the first ataxin shown to predispose to ALS, ${ }^{5}$ with repeat length $\geq 30$ units in European patients with ALS. ${ }^{6,8,12}$ Normal ATXN-2 alleles were in fact $\leq 31$ repeats in several studies., ${ }^{5,6}$ In our sALS cohort from southern Italy, we found a significant association with $A T X N-2$ when PolyQ repeats were $\geq 28$ ( $2.7 \%$ alleles), with only $0.5 \%$ expanded alleles in controls. This makes our finding closer to the study of the American and Chinese ALS cohorts, where the disease was shown to be associated with $A T X N-2$ repeat expansions 27-33 and $\geq 27$, respectively. ${ }^{5,9}$ Furthermore, our study supports 


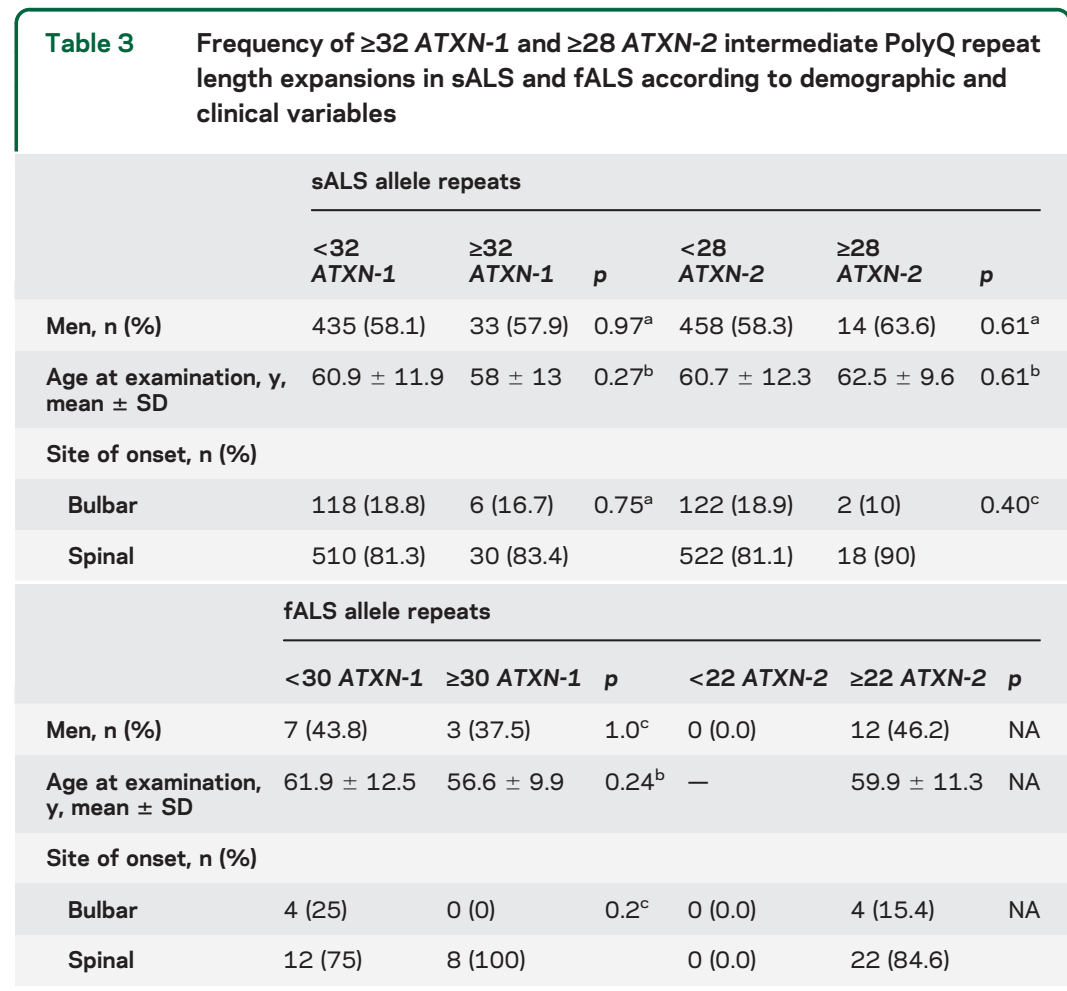

Abbreviations: ATXN = ataxin; fALS = familial amyotrophic lateral sclerosis; NA = not available; PolyQ = polyglutamine; $\mathrm{AALS}=$ sporadic amyotrophic lateral sclerosis.

${ }^{a} \chi^{2}$ test.

${ }^{\mathrm{b}}$ Mann-Whitney $U$ test.

${ }^{c}$ Fisher exact test. detailed analysis of the internal repeat structure further demonstrated that expansions had occurred via at least 2 mechanisms resulting in different internal repeat structures in our carriers (either a single interruption or 2 interruptions).

ATXN-1 PolyQ repeat length intermediate expansions is an emerging genetic risk factor for ALS. ATXN-1 PolyQ repeat expansions are the cause of SCA1, an autosomal dominant spinocerebellar progressive ataxia where signs and symptoms of motor neuron degeneration are reported, and rarely predominate. ${ }^{14,19,23}$ Normal $A T X N-1$ variable CAG repeats ranging from 6 to 44 have been reported in the general population, and those with repeats longer than 20 typically have CAT triplet interruptions within the CAG tract. ${ }^{24}$ In contrast, SCA1-affected alleles have $\geq 39$ repeat expansions and CAT triplet interruptions are lacking. ${ }^{19}$

In our sALS cohort, $7.07 \%$ of the $A T X N-1$ alleles carried repeat expansions $\geq 32$ compared to only $2.4 \%$ of control alleles. ATXN-1 might therefore be a novel risk factor for ALS, and this widens the role of genetic modifiers in ALS. In our cohort, 6 patients carried $A T X N-1$ expanded alleles $(36,37$ repeats) and the sequencing of these revealed the presence of 2 CAT interruptions.

A recent report suggested that several PolyQ genes, including $A T X N-1$, may not be involved in ALS. ${ }^{11}$ In that work, done with American patients with ALS, the ATXN-1 PolyQ repeats expansion range analyzed was $21-37$, with the most common length repeat being 27-28. ${ }^{11}$ Our study showed a different profile; that is, the association with ALS was found with $A T X N-1$ alleles $\geq 32$ repeat lengths, with the most common repeat lengths in both cases and controls being 28-29. The ATXN-1 repeat length variability from population to population and different methodologic approaches might explain the above divergent results. In particular, while Lee et al. ${ }^{11}$ looked at the range of PolyQ repeat lengths both in patients with ALS and controls, we established the best repeat cutoff value to discriminate cases and controls through a ROC analysis.

Analyzing patients with fALS, we found that only $A T X N-1 \geq 30$ PolyQ repeats alleles showed a modestly significant association with the disease. However, as the recruited patients with fALS were relatively few, these results should be replicated in a larger cohort.

We could not find $A T X N-1$ or $A T X N-2$ PolyQ repeat expansion effects on phenotypic variables in our ALS cohort. We could only assess sex, age at onset, and site of onset, and showed that none of them was significantly related to either $A T X N-1$ or $A T X N-2$ PolyQ repeat expansions. Our results are in line with a recent report which showed that ALS phenotype is not affected by $A T X N-2$ PolyQ repeat expansions. ${ }^{10}$ Taken together, both studies argue against a significant 
role for both $A T X N$ repeat expansions as clinical modifiers in ALS.

The demonstration of selective repeat expansions in $A T X N-1$ and $A T X N-2$ in ALS carries significant implications for the understanding of the pathophysiology of this severe neurodegenerative disorder. $A T X N-2$ PolyQ repeats enhance the interaction of ataxin-2 protein with TDP-43, and promote TDP-43 mislocalization into the cytoplasm, altered RNA metabolism, and toxicity to motor neurons. ${ }^{5,25}$ ATXN-1 has RNA-binding activity and can interact with p80 coilin in the nucleoplasm, suggesting a specific role in RNA metabolism. ${ }^{17,18}$ Furthermore, a specific ATXN-1interacting protein, PolyQ binding protein-1 (PQBP1 ), when overexpressed in mice increases ubiquitin nuclear accumulation and induces a progressive motor neuron disease-like phenotype. ${ }^{26}$ Given that the length of PolyQ repeats drives the PQBP-1 affinity to $A T X N-1$, and that this association has been suggested to lead to cell death, ${ }^{27}$ it might indicate that ATXN-1/PQBP-1 aggregates form in motor neurons of patients with ALS bearing $A T X N-1$ repeat length expansions, thus representing a genetic variable that may prompt neurodegeneration.

We further confirm an association between ATXN-2 intermediate expansions and risk for ALS and show that $A T X N-1$ intermediate expansions also play a role as risk factor for the disorder. Both ataxins therefore independently contribute to ALS pathogenesis, probably through a perturbed RNA processing. The identification of the specific roles of ATXN-1 and ATXN-2 PolyQ proteins in motor neuron degeneration and death will give a key contribution for research into pathogenesis of motor neuron diseases.

\section{AUTHOR CONTRIBUTIONS}

Dr. Francesca L. Conforti: designing experiments, statistical analysis, acquisition of data, drafting/revising the manuscript for content. Dr. Rossella Spataro: analysis and interpretation of data, drafting/revising the manuscript. Dr. William Sproviero: acquisition of data, drafting/revising the manuscript. Dr. Rosalucia Mazzei: acquisition of data, analysis and interpretation of data, drafting/revising the manuscript. Dr. Francesca Cavalcanti: acquisition of data, analysis and interpretation of data. Dr. Francesca Condino: statistical analysis, drafting/revising the manuscript for content. Dr. Isabella L. Simone: acquisition of data. Dr. Giancarlo Logroscino: acquisition of data. Dr Alessandra Patitucci: acquisition of data, analysis and interpretation of data, drafting/ revising the manuscript. Dr. Angela Magariello: acquisition of data. Dr. Maria Muglia: acquisition of data, drafting/revising the manuscript. Dr. Carmelo Rodolico: acquisition of data. Dr Paola Valentino: acquisition of data. Dr. Francesco Bono: acquisition of data, analysis and interpretation of data Dr. Tiziana Colletti: acquisition of data. Dr. Maria R. Monsurrò: acquisition of data. Dr. Antonio Gambardella: analysis and interpretation of data, drafting/revising the manuscript. Dr. Vincenzo La Bella: study concept or design, designing experiments, analysis and interpretation of data, drafting/revising the manuscript for content.

\section{ACKNOWLEDGMENT}

The authors thank Martin Russell for revision of the English language.

\section{DISCLOSURE}

The authors report no disclosures relevant to the manuscript. Go to Neurology.org for full disclosures.

Received April 1, 2012. Accepted in final form August 28, 2012.

\section{REFERENCES}

1. Kiernan MC, Vucic S, Cheah BC, et al. Amyotrophic lateral sclerosis. Lancet 2011;377:942-955.

2. Andersen PM, Al-Chalabi A. Clinical genetics of amyotrophic lateral sclerosis: what do we really know? Nat Rev Neurol 2011;7:603-615.

3. Renton AE, Majounie E, Waite A, et al. A hexanucleotide repeat expansion in C9ORF72 is the cause of chromosome 9p21-linked ALS-FTD. Neuron 2011;72:257-268.

4. Dejesus-Hernandez M, Mackenzie IR, Boeve BF, et al. Expanded GGGGCC hexanucleotide repeat in noncoding region of C9ORF72 causes chromosome 9p-linked FTD and ALS. Neuron 2011;72:245-256.

5. Elden AC, Kim H-J, Hart MP, et al. Ataxin-2 intermediate-length polyglutamine expansions are associated with increased risk for ALS. Nature 2010;466:10691075.

6. Lee T, Li Y-R, Ingre C, et al. Ataxin-2 intermediate-length polyglutamine expansions in European ALS patients. Hum Mol Genet 2011;20:1697-1700.

7. Yu Z, Zhu Y, Chen-Plotkin AS, et al. PolyQ repeat expansions in ATXN2 associated with ALS are CAA interrupted repeats. PLoS One 2011;6:e17951.

8. Corrado L, Mazzini L, Oggioni GD, et al. ATXN-2 CAG repeat expansions are interrupted in ALS patients. Hum Genet 2011;130:575-580.

9. Chen YP, Huang R, Yang Y, et al. Ataxin-2 intermediate length polyglutamine: a possible risk factor for Chinese patients with amyotrophic lateral sclerosis. Neurobiol Aging 2011;32:1925.e1-e5.

10. Sorarù G, Clementi M, Forzan M, et al. ALS risk but not phenotype is affected by ataxin- 2 intermediate-length polyglutamine expansion. Neurology 2011;76:2030-2031.

11. Lee T, Li Y-R, Chesi A, et al. Evaluating the prevalence of polyglutamine repeat expansions in amyotrophic lateral sclerosis. Neurology 2011;76:2062-2065.

12. Van Damme P, Veldink JH, van Blitterswijk M, et al. Expanded ATXN2 CAG repeat size in ALS identifies genetic overlap between ALS and SCA2. Neurology 2011;76:2066-2072.

13. Ross OA, Rutherford NJ, Baker M, et al. Ataxin-2 repeatlength variation and neurodegeneration. Hum Mol Gernet 2011;20:3207-3212.

14. Uchihara T, Kobayashi T, Misuzawa H. Predominant motor symptoms in a 74-year-old man with a small elongation in the spinocerebellar atrophy type 1 gene. Neuropathology 2006;26:376-378.

15. Nanetti L, Fancellu R, Tomasello C, et al. Rare association of motor neuron disease and spinocerebellar ataxia type 2 (SCA2): a new case and review of the literature. J Neurol 2009;256:1926-1928.

16. Ohara $S$, Iwahashi $\mathrm{T}$, Oide R, et al. Spinocerebellar ataxia type 6 with motor neuron loss: a follow-up autopsy report. J Neurol 2002;249:633-635.

17. Yue S, Serra HG, Zoghbi HY, Orr HT. The spinocerebellar ataxia type 1 protein, ataxin-1, has RNA-binding activity that is inversely affected by the length of its polyglutamine tract. Hum Mol Genet 2001;10:25-30. 
18. Hong $S$, Ka S, Kim S, et al. p80 coilin, a coiled bodyspecific protein, interacts with ataxin-1, the SCA1 gene product. Biochim Biophys Acta 2003;1638:35-42.

19. Whaley RB, Fujioka S, Wszolek ZK. Autosomal dominant cerebellar ataxia type 1 : a review of the phenotypic and genotypic characteristics. Orphanet J Rare Dis 2011;6:33. e1-e13.

20. Brooks BR, Miller RG, Swash M, Munsat TL. El Escorial revisited: revised criteria for the diagnosis of amyotrophic lateral sclerosis. Amyotroph Lateral Scler Other Motor Neuron Disord 2000;1:293-299.

21. Pulst SM, Nechiporuk A, Nechiporuk T, et al. Moderate expansion of a normally biallelic trinucleotide repeat in spinocerebellar ataxia type 2. Nat Genet 1996;14:269276.

22. Sobczak K, Krzyzosiak WJ. CAG repeats containing CAA interruptions form branched hairpin structures in spinocerebellar ataxia type 2 transcripts. J Biol Chem 2005;280:3898-3910.

23. Sasaki H, Fukazawa T, Yanagihara T, et al. Clinical features and natural history of spinocerebellar ataxia type 1 . Acta Neurol Scand 1996;93:64-71.

24. Chung MY, Ranum LP, Duvick LA, et al. Evidence for a mechanism predisposing to intergenerational CAG repeat instability in spinocerebellar ataxia type I. Nat Genet 1993;5:254-258.

25. Lagier-Tourenne C, Cleveland DW. An expansion in ALS genetics. Nature 2010;466:1052-1053.

26. Okuda T, Hattori $H$, Taskeuchi $S$, et al. PQBP-1 transgenic mice show a late-onset motor neuron disease-like phenotype. Hum Mol Genet 2003;12:711-725.

27. Okazawa $\mathrm{H}$, Rich $\mathrm{T}$, Chang $\mathrm{A}$, et al. Interaction between mutant ataxin-1 and PQBP-1 affects transcription and cell death. Neuron 2002;34:701-713.

\section{Share Your Artistic Expressions in Neurology 'Visions'}

AAN members are urged to submit medically or scientifically related artistic images, such as photographs, photomicrographs, and paintings, to the "Visions" section of Neurology ${ }^{\circledR}$. These images are creative in nature, rather than the medically instructive images published in the Neurolmages section. The image or series of up to six images may be black and white or color and must fit into one published journal page. Accompanying description should be 100 words or less; the title should be a maximum of 96 characters including spaces and punctuation.

Learn more at www.aan.com/view/Visions, or upload a Visions submission at submit.neurology.org.

\section{Call for Submissions: Neurology launches Global Perspectives!}

Section Co-Editors Johan A. Aarli, MD, and Oded Abramsky, MD, PhD, FRCP, encourage submissions to the Global Perspectives section that provides a platform in Neurology for news about scientific findings or academic issues. News may include international research content, spotlights on specific neurologic practice concerns within a country, or important information about international educational or scientific collaborative efforts.

Submissions must be 1,250 words or less with five or less references. A maximum of two figures or two tables (or combination) can be incorporated if necessary. For complete submission requirements, please go to www.neurology.org and click on "Information for Authors." The submissions will be reviewed by the editors and may be edited for clarity.

Interested submitters can register and upload manuscripts under the section "Global Perspectives" at http://submit.neurology.org. Please send inquiries to Kathy Pieper, Managing Editor, Neurology; kpieper@neurology.org. 


\section{Ataxin-1 and ataxin-2 intermediate-length PolyQ expansions in amyotrophic lateral sclerosis}

Francesca L. Conforti, Rossella Spataro, William Sproviero, et al.

Neurology 2012;79;2315; Published online before print November 28, 2012;

DOI 10.1212/WNL.0b013e318278b618

This information is current as of December 11, 2012

Updated Information \&
Services
References
Permissions \& Licensing
Reprints

Updated Information \&

Services

Reprints including high resolution figures, can be found at:

http://www.neurology.org/content/79/24/2315.full.html

This article cites 27 articles, 7 of which can be accessed free at: http://www.neurology.org/content/79/24/2315.full.html\#ref-list $-1$

Information about reproducing this article in parts (figures, tables) or in its entirety can be found online at:

http://www.neurology.org/misc/about.xhtml\#permissions

Information about ordering reprints can be found online: http://www.neurology.org/misc/addir.xhtml\#reprintsus

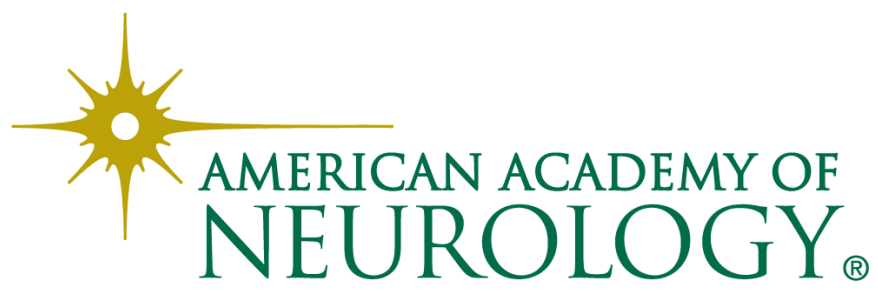

